\title{
Article \\ Analyzing the Neutron Parameters in the Accelerator Driven Subcritical Reactor Using the Mixture of Molten Pb-Bi as Both Target and Coolant
}

\author{
Tien Tran Minh (D)
}

check for updates

Citation: Tran Minh, T. Analyzing the Neutron Parameters in the Accelerator Driven Subcritical Reactor Using the Mixture of Molten $\mathrm{Pb}-\mathrm{Bi}$ as Both Target and Coolant. Atoms 2021, 9, 95. https://doi.org/ 10.3390/atoms 9040095

Received: 7 October 2021

Accepted: 19 November 2021

Published: 21 November 2021

Publisher's Note: MDPI stays neutral with regard to jurisdictional claims in published maps and institutional affiliations.

Copyright: (C) 2021 by the author. Licensee MDPI, Basel, Switzerland. This article is an open access article distributed under the terms and conditions of the Creative Commons Attribution (CC BY) license (https:/ / creativecommons.org/licenses/by/ $4.0 /)$.
Institute of Applied Technology, Thu Dau Mot University, Thu Dau Mot City 820000, Vietnam; tientm@tdmu.edu.vn; Tel.: +84-0907-566-023

\begin{abstract}
In this paper, the Accelerator Driven Subcritical Reactor (ADSR) was simulated based on the structure of the TRIGA-Mark II reactor by the MCNPX program. The proton beam interacts on the $\mathrm{Pb}$-Bi molten target with various energy levels from $0.5 \mathrm{GeV}$ to $2.0 \mathrm{GeV}$. The important neutron parameters to evaluate the operability of ADSR were calculated as: the neutron yields according to various thicknesses of the target and according to the energy of the incident proton beam; the effective neutron multiplication factor for various fuel mixtures, along with its stability for some fuel mixtures; the axial and radial distributions of the neutron flux along with the height and radius of the core. The obtained results had shown a good agreement in using $\mathrm{Pb}-\mathrm{Bi}$ molten as the interaction target and coolant for ADSR.
\end{abstract}

Keywords: ADSR; molten Pb-Bi; neutron yield; effective multiplication factor; neutron flux

\section{Introduction}

The Accelerator-Driven Subcritical Reactor (ADSR) is a great research interest in the world since it was suggested back by many authors, such as C. Rubbia; K. Furukawa, C. D. Bowman et al. [1-3]. It can generate energy and transmute radioactive wastes cleanly and safely. The recent researches about ADSR concentrate on neutron source, designing target, and fuel.

Regarding interactive targets, the most popular studies are using solid targets to generate neutrons. In 1999, the group of authors X. Ledoux, F. Borne, A. Boudard, et al. calculated the energy spectrum of neutrons emitted at different angles when a proton beam interacts on the lead target with energies of $0.8 \mathrm{MeV}, 1.2 \mathrm{MeV}$, and 1.6 MeV respectively [4]. Also in 1999, the group of authors S. Meigo et al. calculated the distribution of the neutron flux from a thick lead target with incident proton energies of $0.5 \mathrm{GeV}$ and $1.5 \mathrm{GeV}$ by the MCNP4A program [5].

Commonly used coolants are light water, molten salt, or molten $\mathrm{Pb}-\mathrm{Bi}$ mixture. The group of authors Hasanzadeh et al. used the MCNP program to perform simulation of Moroccan TRIGA-Mark II Reactor and calculate Neutrons Kinetic and Parameters [6,7]. In these studies, the coolant was light water. Also from this model, Abdelaziz Darif et al. [8] calculated the dependence of the effective neutron multiplication factor $\left(\mathrm{k}_{\mathrm{eff}}\right)$ on the number of reflectors in the core of the reactor. The results showed that keff can be increased without changing the size of the core. These authors also analyze neutron parameters using DRAGON.v5 and TRIVAC.v5 codes [9]. Ned Xoubi researched the neutron source for Jordan's subcritical reactor [10]. In this studying, a proton is coupled to a subcritical assembly with a lead target; the spallation neutron source is investigated; 3-D Monte Carlo simulating of radiation transport is performed for the system. These results had shown an increase of seven orders of magnitude compared to the current JSA Pu-Be sourcedriven core. Xue-Chao Zhao et al. conducted an analysis of Th-U breeding capability for an accelerator-driven subcritical molten salt reactor [11]. These results had shown the 
subcriticality of the core has a considerable effect on the Th-U breeding; a high subcriticality is favorable to improving the conversion ratio, increasing the net U-235 production, and reducing the doubling time.

There have been many proposals and calculations using liquid lead as an interactive target and coolant [12-17].

In general, there are not many studies on neutron parameters for ADSR using molten $\mathrm{Pb}-\mathrm{Bi}$ as a target and coolant. In this paper, ADSR was simulated based on TRIGA-Mark II reactor structure [6-8] using the MCNPX program. From this structure, the neutron parameters were calculated, including:

- The neutron yields on molten $\mathrm{Pb}$-Bi target;

- The effective neutron multiplication factors $\mathrm{k}_{\mathrm{eff}}$;

- The distributions of the neutron fluxes along the height of ADSR with molten Pb-Bi target, U-Th mixed fuels;

- The distributions of the neutron fluxes along a radial of ADSR with molten $\mathrm{Pb}-\mathrm{Bi}$ target, U-Th mixture fuels.

\section{Model and Calculations}

The TRIGA-Mark II subcritical reactor model was simulated as the Figure 1. Some characteristics of the core are shown in Table 1. The structure of the core consists of 108 fuel rods. The core is divided into 7 rings. All of these are assumed to be placed in the molten lead-bismuth medium.

Table 1. Structure of the core of the TRIGA- Mark II reactor.

\begin{tabular}{cc}
\hline Characteristics & Details \\
\hline Fuels & $\mathrm{UThO}^{-3}$ \\
Density of the fuel & $10.32 \mathrm{~g} \mathrm{~cm}^{-3}$ \\
Coolant & Molten lead-bismuth \\
Reflectors & Graphite \\
Density of the reflectors & $2.25 \mathrm{~g} \mathrm{~cm}^{-3}$ \\
Control rods & $\mathrm{B}_{4} \mathrm{C}$ \\
Diameter of the fuels & $3.73 \mathrm{~cm}$ \\
Height of the fuels & $38.1 \mathrm{~cm}$ \\
Diameter of the core & $56 \mathrm{~cm}$ \\
Height of the core & $72 \mathrm{~cm}$ \\
\hline
\end{tabular}

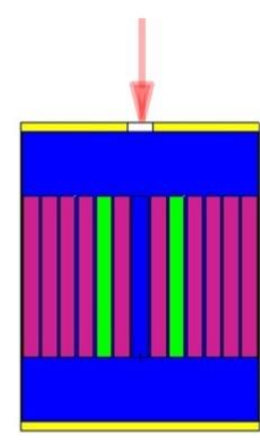

Fuel rods

Graphites

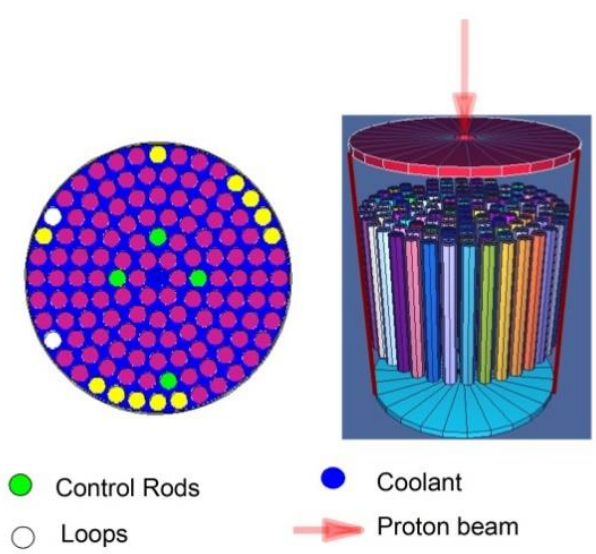

Figure 1. Structure of the TRIGA-Mark II core by MCNP code.

From this structure, the neutron parameters were calculated. The results were presented below. 


\subsection{The Neutron Yields $\left(Y_{n / p}\right)$ from the Molten Pb-Bi Target}

The proton beam from the accelerator interacts with the molten Pb-Bi target. From there, the neutron yields were calculated. The MCNPX program was used in this case. The Bertini model in MCNPX was chosen for the calculation because it is the most suitable model according to previous studies $[18,19]$. The interacting target was molten $\mathrm{Pb}-\mathrm{Bi}$ consisting of isotopes $\mathrm{Pb}-204, \mathrm{~Pb}-206, \mathrm{~Pb}-207, \mathrm{~Pb}-208$ with ratios of $1.4 \%, 24.1 \%, 22.1 \%$, $52.4 \%$, respectively. The molten Pb-Bi mixture consists of $44.5 \%$ lead and $55.5 \%$ bismuth.

- The neutron yields according to target thicknesses

In this section, the incident proton with $1 \mathrm{GeV}$ energy interacts with the molten $\mathrm{Pb}-\mathrm{Bi}$ target. From here, the neutron yields were calculated with different target thicknesses from $10 \mathrm{~cm}$ to $50 \mathrm{~cm}$. These results were compared with previous works [20,21]. The authors Giao N. M. et al. [20] calculated the neutron yield by using data from the Japanese library JENDL-HE. Mariono et al. [21] performed calculations based on the Bertini model. These results were presented in Table 2 and Figure 2.

Table 2. The neutron yields from molten $\mathrm{Pb}$-Bi target with different thicknesses from $10 \mathrm{~cm}$ to $50 \mathrm{~cm}$.

\begin{tabular}{cccc}
\hline \multirow{2}{*}{ Thickness (cm) } & \multicolumn{3}{c}{ Neutron Yield (n/p) } \\
\cline { 2 - 4 } & Pb-Solid [14] & Pb-Solid [15] & Pb-Bi-Liquid (This Work) \\
\hline 10 & 10.968 & 9.080 & 9.899 \\
20 & 18.852 & 16.087 & 17.616 \\
30 & 22.215 & 20.859 & 22.839 \\
40 & 23.926 & 23.480 & 25.862 \\
50 & 24.856 & 24.954 & 27.561 \\
\hline
\end{tabular}

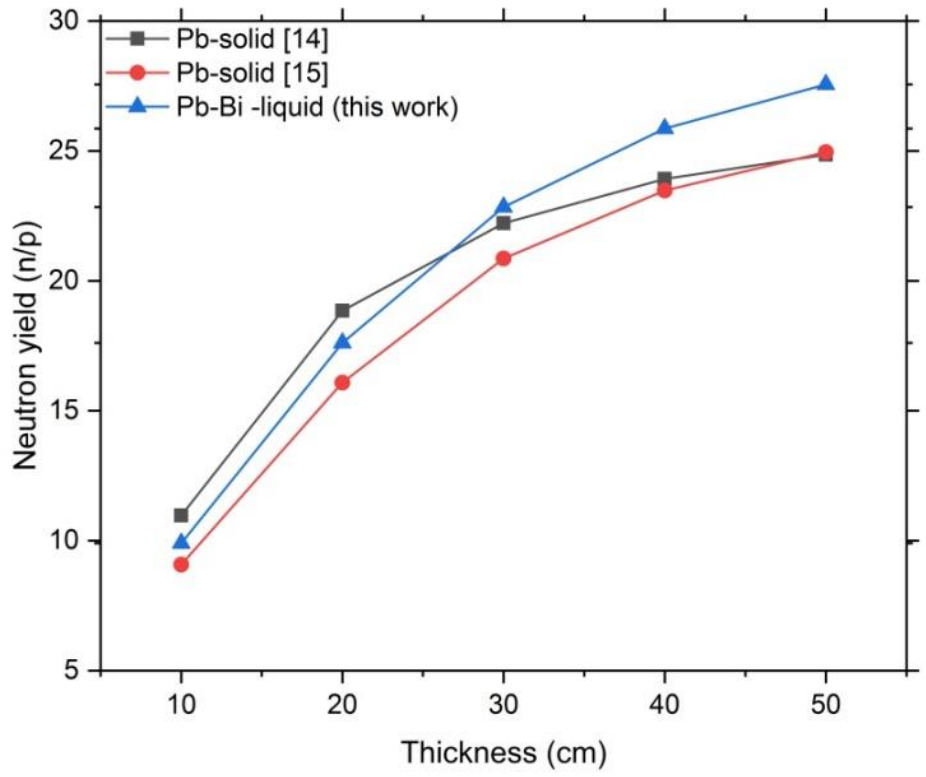

Figure 2. The neutron yields from molten $\mathrm{Pb}-\mathrm{Bi}$ target with different thicknesses from $10 \mathrm{~cm}$ to $50 \mathrm{~cm}$.

These results showed that the neutron yields on the molten Pb-Bi target by thickness were similar to that for molten lead. When the target thickness was $10 \mathrm{~cm}$, the medium neutron yield was about $10 \mathrm{n} / \mathrm{p}$. As the thickness increases, $Y_{n / p}$ also increases; at the $50 \mathrm{~cm}$ thickness, $Y_{n / p}$ from $25 \mathrm{n} / \mathrm{p}$ to $27 \mathrm{n} / \mathrm{p}$. $\mathrm{Y}_{\mathrm{n} / \mathrm{p}}$ for solid $\mathrm{Pb}-\mathrm{Bi}$ was lower than that calculated by the authors N. M. Giao [20] et al. with thicknesses from $10 \mathrm{~cm}$ to $27 \mathrm{~cm}$ but higher from $27 \mathrm{~cm}$ to $50 \mathrm{~cm}$. The difference was highest at $10 \mathrm{~cm}$ thickness with $10.8 \%$ and lowest at $30 \mathrm{~cm}$ thick with $2.7 \%$. $\mathrm{Y}_{\mathrm{n} / \mathrm{p}}$ for liquid $\mathrm{Pb}-\mathrm{Bi}$ compared with the study of Maiorino et al. [19] on the solid lead target is always higher at all thicknesses. The highest difference was at the thickness of $50 \mathrm{~cm}$ with $9.5 \%$ and the lowest at the thickness of $10 \mathrm{~cm}$ 
with $8.3 \%$. With these results, the use of $\mathrm{Pb}$-Bi-liquid as a neutron-generating target in ADSR is completely appropriate. Based on these data, the designing of the ADSR core can be conducted to the requirements of each system.

- The neutron yields according to the levels of energy of the incident proton beam

In these calculations, the proton beam had energies from $0.5 \mathrm{GeV}$ to $2.0 \mathrm{GeV}$. The length of the target was given to be equivalent to the height of the reactor core, $35 \mathrm{~cm}$. These results were presented in Table 3 and Figure 3.

Table 3. The neutron yields from the proton beam with the levels of energy from $0.5 \mathrm{GeV}$ to $2.0 \mathrm{GeV}$.

\begin{tabular}{cccc}
\hline \multirow{2}{*}{ Proton Energy $(\mathrm{GeV})$} & \multicolumn{3}{c}{ Neutron Yield (n/p) } \\
\cline { 2 - 4 } & $\mathbf{P b}$ (Solid) & $\mathbf{P b}$ (Liquid) & Pb-Bi (Liquid) \\
\hline 0.5 & 8.3540 & 8.5310 & 9.7400 \\
0.8 & 16.896 & 17.035 & 19.193 \\
1.0 & 21.952 & 21.968 & 24.526 \\
1.5 & 32.804 & 32.758 & 36.569 \\
2.0 & 42.595 & 42.192 & 46.909 \\
\hline
\end{tabular}

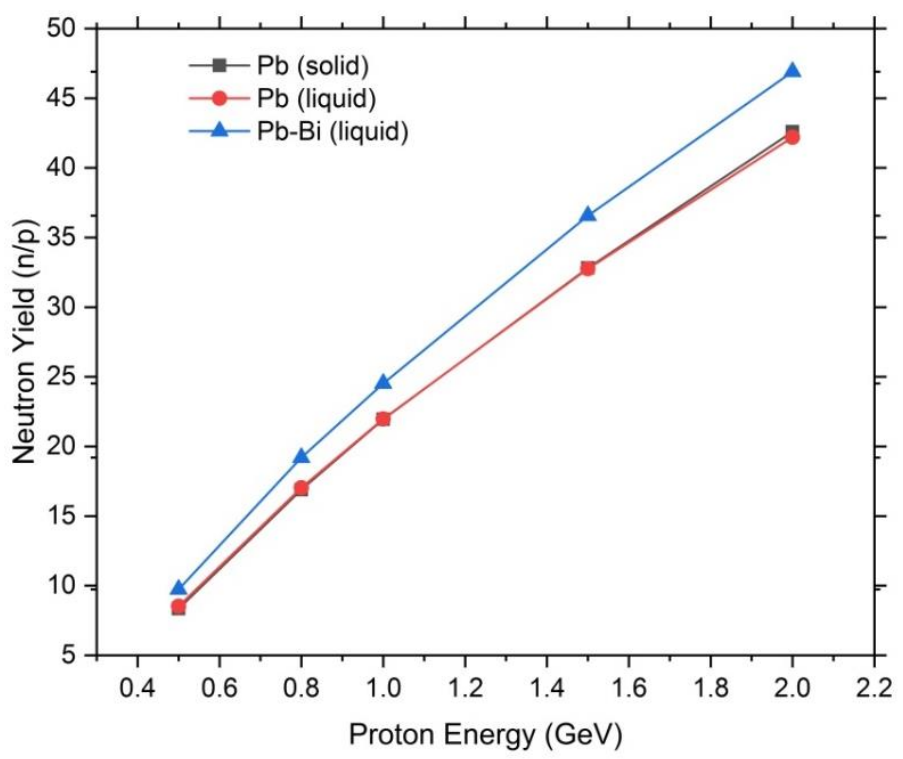

Figure 3. The neutron yields from the proton beam with the levels of energy from $0.5 \mathrm{GeV}$ to $2.0 \mathrm{GeV}$.

The results have shown that the neutron yield from molten $\mathrm{Pb}$-Bi target increases with the incident proton beam intensity, similar to the case of solid lead and liquid lead targets. However, $\mathrm{Y}_{\mathrm{n} / \mathrm{p}}$ for the molten $\mathrm{Pb}-\mathrm{Bi}$ was always higher than all with energies of the incident proton beam, from $9.2 \%$ to $14.2 \%$. The highest difference is when compared with the case of proton beam intensity of $0.5 \mathrm{GeV}$, on the liquid lead target, with $14.2 \%$. The lowest difference was when compared with the case of proton beam intensity of $2.0 \mathrm{GeV}$, on the liquid lead target, with $9.2 \%$. These results further confirmed the possibility of using molten Pb-Bi for ADSR.

\subsection{The Effective Neutron Multiplication Factors $k_{\text {eff }}$}

For ADSR can operate and generate positive energy, the effective neutron multiplication factor $k_{\text {eff }}$ must be greater than 0.68 [21]. In this section, the neutron was investigated for ADSR using the molten $\mathrm{Pb}-\mathrm{Bi}$ and compared with the molten $\mathrm{Pb}$ case. The fuels used were a mixture of Th-UO with different component ratios. The stability of $k_{\text {eff }}$ in each case was also investigated.

- Calculating $\mathrm{k}_{\text {eff }}$ factor 
The investigated Th-UO fuel mixture consists of 8 cases, numbered from F1 to F8, according to the increasing proportion of uranium and decreasing of thorium. Details of fuel composition ratio and the results of medium keff calculation were presented in Table 4 .

Table 4. Details of the ratio of components in the fuel mixture and the results of $k_{\text {eff. }}$

\begin{tabular}{cccccc}
\hline Fuel & ${ }_{90}^{232} \mathbf{T h}$ & ${ }_{92}^{233} \mathbf{U}$ & ${ }_{8}^{16} \mathbf{O}$ & $\mathbf{k}_{\text {eff }}(\mathbf{P b})$ & $\mathbf{k}_{\text {eff }}(\mathbf{P b}-\mathbf{B i})$ \\
\hline F1 & 0.800 & 0.100 & 0.100 & 0.45345 & 0.430634 \\
F2 & 0.700 & 0.200 & 0.100 & 0.71546 & 0.708338 \\
F3 & 0.610 & 0.290 & 0.100 & 0.90719 & 0.902307 \\
F4 & 0.600 & 0.300 & 0.100 & 0.92764 & 0.919357 \\
F5 & 0.580 & 0.320 & 0.100 & 0.96123 & 0.959649 \\
F6 & 0.570 & 0.330 & 0.100 & 0.97984 & 0.974404 \\
F7 & 0.565 & 0.335 & 0.100 & $\mathbf{0 . 9 8 7 5 1}$ & $\mathbf{0 . 9 7 9 4 3 4}$ \\
F8 & 0.560 & 0.340 & 0.100 & $\mathbf{1 . 0 0 0 4 4}$ & $\mathbf{0 . 9 8 3 2 6 0}$ \\
\hline
\end{tabular}

The results have shown that, as the uranium ratio increased, the thorium ratio decreased, the keff increased. With F1 fuel, the keff for both $\mathrm{Pb}$ and $\mathrm{Pb}-\mathrm{Bi}$ was low, less than 0.5. From F2 fuel, keff reaches a value higher than 0.68 . The $\mathrm{k}_{\mathrm{eff}}$ for molten $\mathrm{Pb}$ and $\mathrm{Pb}-\mathrm{Bi}$ differs on average by 0.01 with the fuel mixtures. However, with the F 8 fuel mixture, the average $\mathrm{k}_{\text {eff }}$ was greater than 1.0 for $\mathrm{Pb}$, but for $\mathrm{Pb}-\mathrm{Bi}$ it is still less than 1.0. These should be considered carefully because, with $\mathrm{Pb}$, the reactor has reached the critical state, while with $\mathrm{Pb}-\mathrm{Bi}$, it is still in the subcritical state.

- $\quad$ Stability of $\mathrm{k}_{\mathrm{eff}}$ in some fuel mixtures

From the results above, some fuel mixtures were selected to evaluate the stability of the keff in some cycles. The selected mixtures were F2, F3, F5, and F7. With these mixtures, the ADSR generates positive energy and operates in a subcritical state. Critical calculations skipped the first 100 cycles, selects 16 keff values of cycles from 100 to 250 to evaluate. The results were shown in Figure 4.

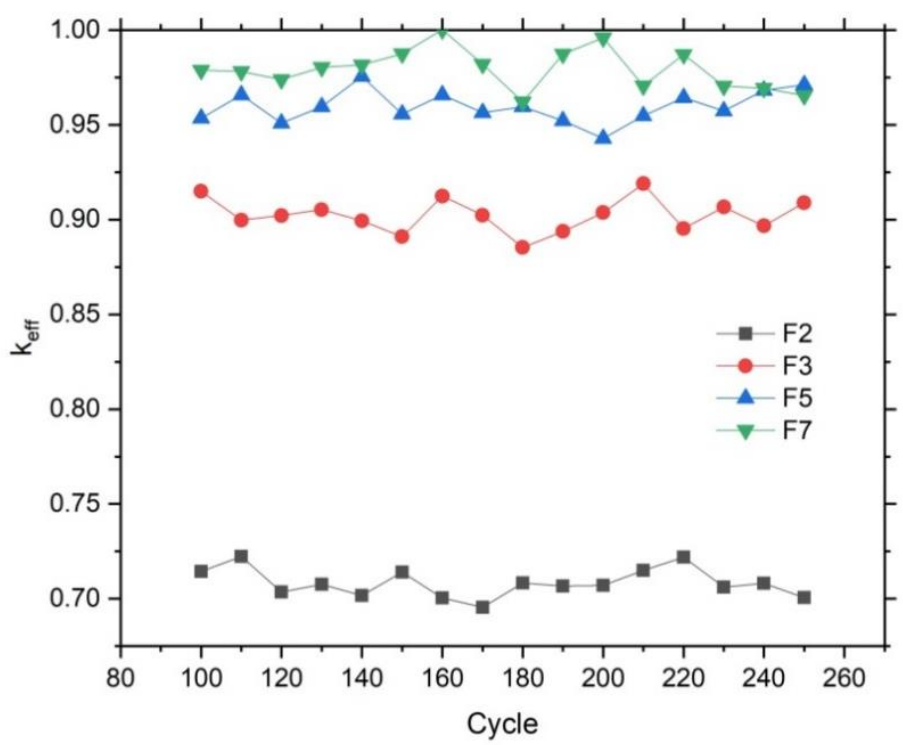

Figure 4. The $\mathrm{k}_{\mathrm{eff}}$ from 100 to 250 cycles.

These results have shown that the values of $k_{\text {eff }}$ were relatively stable throughout all cycles. For the case of the F2 fuel, the medium $\mathrm{k}_{\text {eff }}$ is 0.708338 then the range is from 0.69541 to 0.72226 . With the F3 fuel, the medium $k_{\text {eff }}$ is 0.902307 , the values vary from 0.88528 to 0.91899 . In the case of the F5 fuel, the value of $\mathrm{k}_{\text {eff }}$ is 0.902307 , ranging from 0.94279 to 0.97565. With the F7 fuel, the mean $\mathrm{k}_{\text {eff }}$ is 0.979434 and ranges from 0.9621 to 1.00029 . In 
particular, for the case of the F7 fuel, the maximum of $\mathrm{k}_{\mathrm{eff}}$ is sometimes greater than 1.0. Therefore, careful studies and calculations are needed if using this fuel at this ratio.

\subsection{The Radial and Axial Distributions of the Neutron Fluxes}

In this section, the neutron fluxes were calculated using the F2, F3, F5, F7 fuel mixtures. The MCNP5 program and F4mesh tally were used. The neutron fluxes were calculated from the F4mesh tally in MCNPX based on Equation (1):

$$
\mathrm{F} 4=\mathrm{C} \int \Phi(\mathrm{E}) \mathrm{R}(\mathrm{E}) \mathrm{dE}
$$

with normalization for the current of proton beam I (mA) and neutron yield $Y_{n / p}$. Where $C$ is a multiplication, $\left(\mathrm{ncm}^{-2} \mathrm{~s}^{-1}\right)$ is the neutron flux and $\mathrm{R}(\mathrm{E})$ is any combination of sums and products of energy-dependent quantities known to MCNPX [22]. The neutron fluxes have been determined according to Equation (2) [7]:

$$
\Phi=\frac{2 \times 10^{-3} \mathrm{C} / \mathrm{s}}{\mathrm{mA}} \times \frac{1 \mathrm{p}}{1.6 \times 10^{19} \mathrm{C}} \times \mathrm{F} 4 \times \mathrm{Y}_{\mathrm{n} / \mathrm{p}}
$$

with $Y_{n / p}(n / p)$ is the neutron yield.

- The axial distributions of the neutron fluxes

The neutron fluxes were calculated at 20 locations along with the height of the fuel rod inside the core. The results were shown in Figure 5.

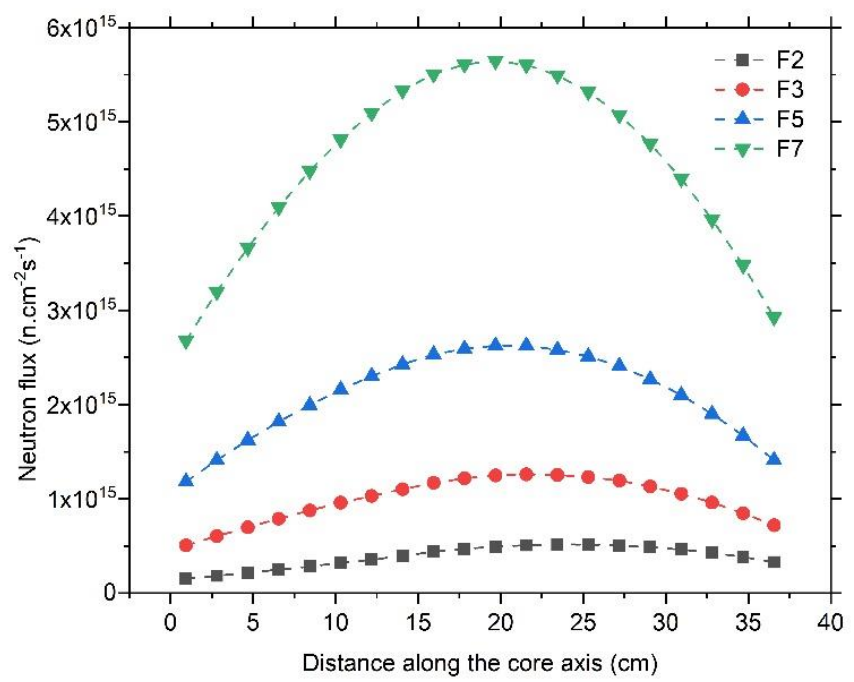

Figure 5. The axial distributions of the neutron fluxes with molten $\mathrm{Pb}$-Bi target.

These results have shown that the distributions of the neutron fluxes with different fuel cases have the same form. The neutron flux was maximum value in the center of the core and decreases towards the sides. As the proportion of uranium in the mixture increased, as the proportion of thorium decreased, the maximum neutron flux increased. However, the exact maximum locations of the neutron flux were also varying. With the F2 fuel, the maximum flux was $5.15903 \times 10^{14}\left(\mathrm{ncm}^{-2} \mathrm{~s}^{-1}\right)$ at position $\mathrm{h}=23.438 \mathrm{~cm}$; with the F3 fuel, the maximum flux was $1.25981 \times 10^{15}\left(\mathrm{ncm}^{-2} \mathrm{~s}^{-1}\right)$ at position $\mathrm{h}=21.562 \mathrm{~cm}$. Particularly for the two cases F5 and F7, the maximum flux position was the same, at the same position $17.812 \mathrm{~cm}$, with the maximum flux being $2.62486 \times 10^{15}\left(\mathrm{ncm}^{-2} \mathrm{~s}^{-1}\right)$ and $5.6457 \times 10^{15}\left(\mathrm{ncm}^{-2} \mathrm{~s}^{-1}\right)$, respectively. These results had shown that the greater the percentage of uranium, the closer the position of the maximum neutron flux was to the center of the core.

- The radial distributions of the neutron fluxes 
The neutron fluxes were calculated at 20 locations along the radius of the core. These results were shown in Figure 6.

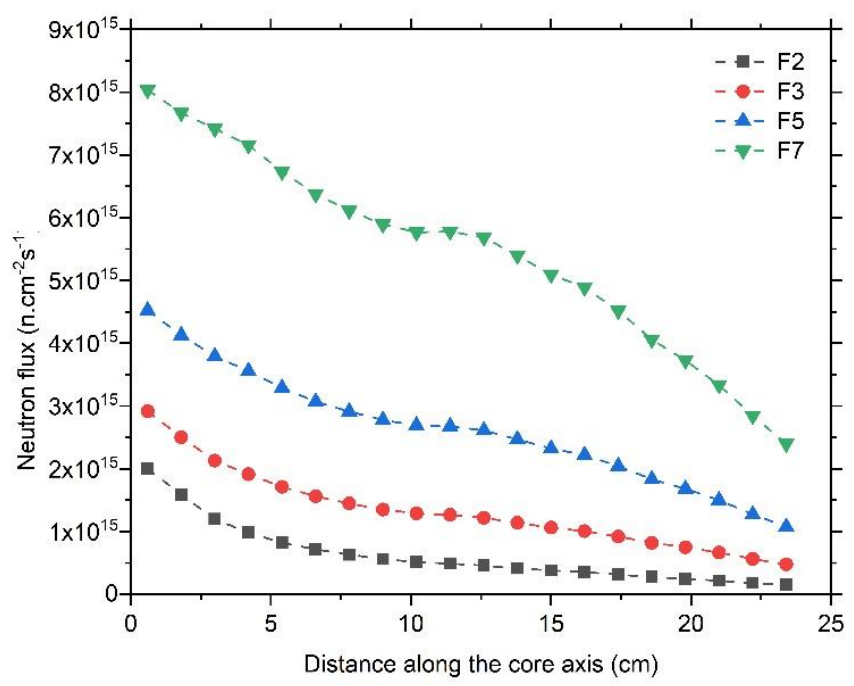

Figure 6. The radial distributions of the neutron flux with molten $\mathrm{Pb}-\mathrm{Bi}$ target.

These results also showed that the maximum neutron flux at the center of the core and gradually decreases to the outside. As the proportion of uranium in the mixture increased, the maximum neutron flux increased. With the F7 fuel mixture, the maximum neutron flux reached more than $8 \times 10^{15}\left(\mathrm{ncm}^{-2} \mathrm{~s}^{-1}\right)$. The neutron fluxes decreased rapidly from the center of the core to a radius of $8 \mathrm{~cm}$, then it began to decrease slowly, and from $8 \mathrm{~cm}$ to $12 \mathrm{~cm}$, the neutron fluxes were almost unchanged. From $12 \mathrm{~cm}$, the neutron fluxes continued to decrease but slower than at the beginning.

These results showed that the neutron fluxes for the molten $\mathrm{Pb}$-Bi target using the mixed fuel cases F2, F3, F5, F7 were very high and suitable for the operation of ADSR. Depending on the actual conditions, requirements, and operational purposes of each ADSR, it is necessary to choose the appropriate fuel component ratio.

\section{Conclusions}

In this paper, the ADSR was simulated based on the structure of the TRIGA-Mark II reactor. From this structure, the neutron parameters have been calculated by MCNPX and MCNP5 programs. Here, the molten $\mathrm{Pb}-\mathrm{Bi}$ mixtures were used as both a target and coolant. The results included the neutron yield on molten lead and molten $\mathrm{Pb}$ - $\mathrm{Bi}$ targets; the effective neutron multiplication factor $\left(k_{\text {eff }}\right)$ with different ratios of fuel mixtures containing thorium and uranium; evaluate the stability of the $k_{\text {eff }}$ in several fuel mixtures over several cycles; calculated the radial and axial distribution of the neutron fluxes. The results have shown that Molten Pb-Bi was very suitable to be used both as an interactive target and as a coolant in ADSR. There are only a few remarks about the proportions of uranium in the mixture that needs to be carefully calculated so that the ADSR generates positive energy and remains subcritical. These results contribute more data for the researching and designing of ADSR most effectively and economically.

Funding: This research received no external funding.

Institutional Review Board Statement: Not applicable.

Informed Consent Statement: Not applicable.

Data Availability Statement: Not applicable.

Conflicts of Interest: The author declare that there is no conflict of interests regarding the publication of this paper. 


\section{References}

1. Roche, C.; Revol, J.P.; Mandrillon, P.; Klapisch, R.; Kadi, Y.; Gelès, C.; Galvez, J.; Fiétier, N.; Carminati, F.; Buono, S.; et al. Conceptual Design of a Fast Neutron Operated High Power Energy Amplifier (No. CERN-AT-95-44-ET); Los Alamos National Laboratory: Los Alamos, NM, USA, 2005.

2. Furukawa, K.; Kato, Y.; Ohmichi, T.; Ohno, H. Combined system of accelerator molten-salt breeder (AMSB) apd molten-salt converter reactor (MSCR). At. Tekhnika Rub. 1983, 15, 23-29.

3. Bowman, C.; Arthur, E.; Lisowski, P.; Lawrence, G.; Jensen, R.; Anderson, J.; Blind, B.; Cappiello, M.; Davidson, J.; England, T.; et al. Nuclear energy generation and waste transmutation using an accelerator-driven intense thermal neutron source. Nucl. Instrum. Methods Phys. Res. Sect. A Accel. Spectrometers Detect. Assoc. Equip. 1992, 320, 336-367. [CrossRef]

4. LeDoux, X.; Borne, F.; Boudard, A.; Brochard, F.; Crespin, S.; Drake, D.; Duchazeaubeneix, J.C.; Durand, D.; Durand, J.M.; Fréhaut, J.; et al. Spallation Neutron Production by 0.8, 1.2, and 1.6 GeV Protons on Pb Targets. Phys. Rev. Lett. 1999, 82, 4412. [CrossRef]

5. Meigo, S.; Takada, H.; Chiba, S.; Nakamoto, T.; Ishibashi, K.; Matsufuji, N.; Maehata, K.; Shigyo, N.; Watanabe, Y.; Numajiri, M. Measurements of neutron spectra produced from a thick lead target bombarded with 0.5- and 1.5-GeV protons. Nucl. Instrum. Methods Phys. Res. Sect. A Accel. Spectrometers Detect. Assoc. Equip. 1999, 431, 521-530. [CrossRef]

6. Hassanzadeh, M. Optimization and analysis of the effects of physical parameters in a TRIGA-ADSR. Kerntechnik 2017, 82, 334-338. [CrossRef]

7. Hassanzadeh, M.; Feghhi, S.A.H. Sensitivity analysis of core neutronic parameters in accelerator driven subcritical reactors. Ann. Nucl. Energy 2014, 63, 228-232. [CrossRef]

8. Darif, A.; Chetaine, A.; Mghar, M.; Kabach, O. Investigation of the influence of various reflector elements material types on the core nuclear parameters of the 2 MW Triga Mark-II research reactor using the Monte Carlo code. Turk. J. Phys. 2018, 42, 41-46. [CrossRef]

9. Darif, A.; Chetaine, A.; Kabach, O.; Mghar, M.; Saidi, A. Neutronic Analysis of Moroccan TRIGA MARK-II Research Reactor using the DRAGON. v5 and TRIVAC. v5 codes. Phys. AUC 2017, 27, 41-49.

10. Xoubi, N. Neutronic design study of accelerator driven system (ADS) for Jordan subcritical reactor as a neutron source for nuclear research. Appl. Radiat. Isot. 2018, 131, 71-76. [CrossRef] [PubMed]

11. Zhao, X.-C.; Cui, D.-Y.; Cai, X.-Z.; Chen, J.-G. Analysis of Th-U breeding capability for an accelerator-driven subcritical molten salt reactor. Nucl. Sci. Tech. 2018, 29, 121. [CrossRef]

12. Tien, T.M. Distributions of neutron flux from $(\mathrm{p}, \mathrm{n})$ reaction on the liquid lead target for accelerator driven subcritical reactor (ADSR). J. Phys. Conf. Ser. 2019, 1324, 012061. [CrossRef]

13. Tien, T.M. Distribution of Neutrons from the Reaction $(\mathrm{p}, \mathrm{n})$ on the Liquid Lead Target in the Accelerator Driven System Reactor. J. Phys. Conf. Ser. 2019, 1172, 012066. [CrossRef]

14. Tien, T.M.; Dung, T.Q. Calculation of the neutron flux distribution in the accelerator driven subcritical reactor with (Th-233U) $\mathrm{O}_{2}$ and (Th-235U) $\mathrm{O}_{2}$ mix fuel. J. Phys. Conf. Ser. 2020, 1451, 012009. [CrossRef]

15. Tien, T.M.; Khanh, N.K.; Hien, B.T.T.; Luong, N.T.T.; Phung, N.H.T.; Thi, N.T.M. K effective factor in the ADSR using liquid lead target and (Th233U) $\mathrm{O}_{2}$, (Th235UO) 2 , (Th238U) $\mathrm{O}_{2}$ fuel mixture. J. Phys. Conf. Ser. 2020, 1706, 012009. [CrossRef]

16. Tien, T.M.; Khanh, N.K.; Ngan, N.K.; Nhi, N.T.T. Radioactive decay of thorium and uranium in the liquid lead and molten salt. IOP Conf. Ser. Mater. Sci. Eng. 2021, 1070, 012024. [CrossRef]

17. Tien, T.M.; Phung, N.H.T.; Hien, B.T.T. Effect of reflector materials to the neutron flux and k effective in the accelerator driven subcritical reactor. IOP Conf. Ser. Mater. Sci. Eng. 2021, 1070, 012025. [CrossRef]

18. İskender, D.; Arasoğlu, A.; Tel, E. Neutron multiplicity with 1.0 and $1.2 \mathrm{GeV}$ proton-induced spallation reactions on thin targets. Chin. J. Phys. 2008, 46, 124-134.

19. Maiorino; Jose, R.; Mongelli, S.T.; Santos, A.; Anefalos, S.; Deppman, A.; Carluccio, T. A review of models and codes for neutron source (spallation) calculation for ADS application. In Proceedings of the International Nuclear Atlantic Conference-INAC 2005, Santos, Brazil, 28 August-2 September 2005; p. 85-99141-01-5.

20. Nguyen; Giao, M.; Le Thi, T.T.; Nguyen, T.A.T. Screening Effect in (pn) Reactions on Heavy Element Targets 82206 Pb, 92238 U, 74184 W, 79197 Au. No. IC-2010/057. Abdus Salam Int. Cent. Theor. Phys. 2010, 43, 43048729.

21. Nifenecker, H.; David, S.; Loiseaux, J.M.; Meplan, O. Basics of accelerator driven subcritical reactors. Nucl. Instrum. Methods Phys. Res. Sect. A Accel. Spectrometers Detect. Assoc. Equip. 2001, 463, 428-467. [CrossRef]

22. Pelowitz, Desise B. “MCNPX User's Manual, Version 2.5.0, April 2005.” LACP05-0369, 2005. Available online: https:/ /www.osti. gov/etdeweb/servlets/purl/602571 (accessed on 21 August 2021). 